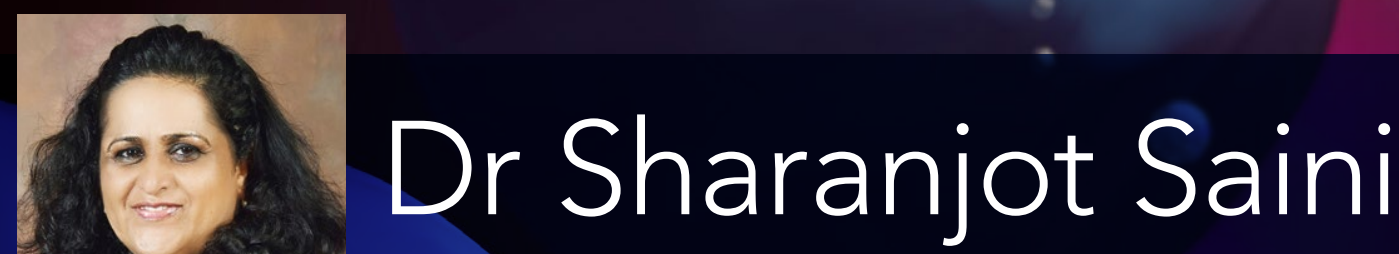

E: ssaini@augusta.edu T: +17067210856

W: web2.augusta.edu/faculty/directory/view.php?id=SSAINI

\title{
Exosome microRNAs as liquid biopsies for the monitoring of prostate cancer
}

\section{Research Objectives}

Dr Saini's lab focuses on harnessing the potential of exosomes as a source of novel cancer biomarkers and for engineering novel therapies against prostate cancer.

\section{Detail}

\section{Address}

Sharanjot Saini

1410 Laney Walker Blvd

Augusta, GA, 30912, USA

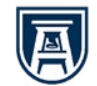

\section{AUGUSTA \\ UNIVERSITY}

\section{Bio}

Dr Saini is an Associate Professor based at the Medical College of Georgia at Augusta University. Her lab focuses on studying the mechanistic basis of aggressive prostate cancer, with a long-term goal of identifying novel biomarkers and targeted therapies against advanced disease.

\section{Funding}

- Department of Defense (W81XWH-1-0303)

- National Institutes of Health (Grant Number RO1CA177984).

\section{Collaborators}

- Dr Barbara Bensing

- Dr Ashok Sharma

- Dr Nikhil Patel

\section{References}

Bhagirath, D, Liston, M, Akoto, T, et al, (2021) Novel, non-invasive markers for detecting therapy induced neuroendocrine differentiation in castration-resistant prostate cancer patients. Scientific reports, 11(1), 8279. doi.org/10.1038/s41598-021-87441-2

Bhagirath, D, Liston, M, Patel, N, et al, (2020) MicroRNA determinants of neuroendocrine differentiation in metastatic castration-resistant prostate cancer. Oncogene, 39(49), 7209-7223. doi.org/10.1038/s41388-020-01493-8

\section{Personal Response}

When do you think your miRNA-based classifier system will be available as a widely available diagnostic tool?

IV For the miRNA-based classifier to be available as a diagnostic tool, future validation studies with larger clinical cohorts are warranted. We believe that upon further validation, these markers can be translated to the clinic for diagnosing NED in CRPC patients and predicting response to androgen pathway inhibitors that is currently challenging. 
Health \& Medicine | Sharanjot Saini

\section{Exosome microRNAs as liquid biopsies for the monitoring of prostate cancer}

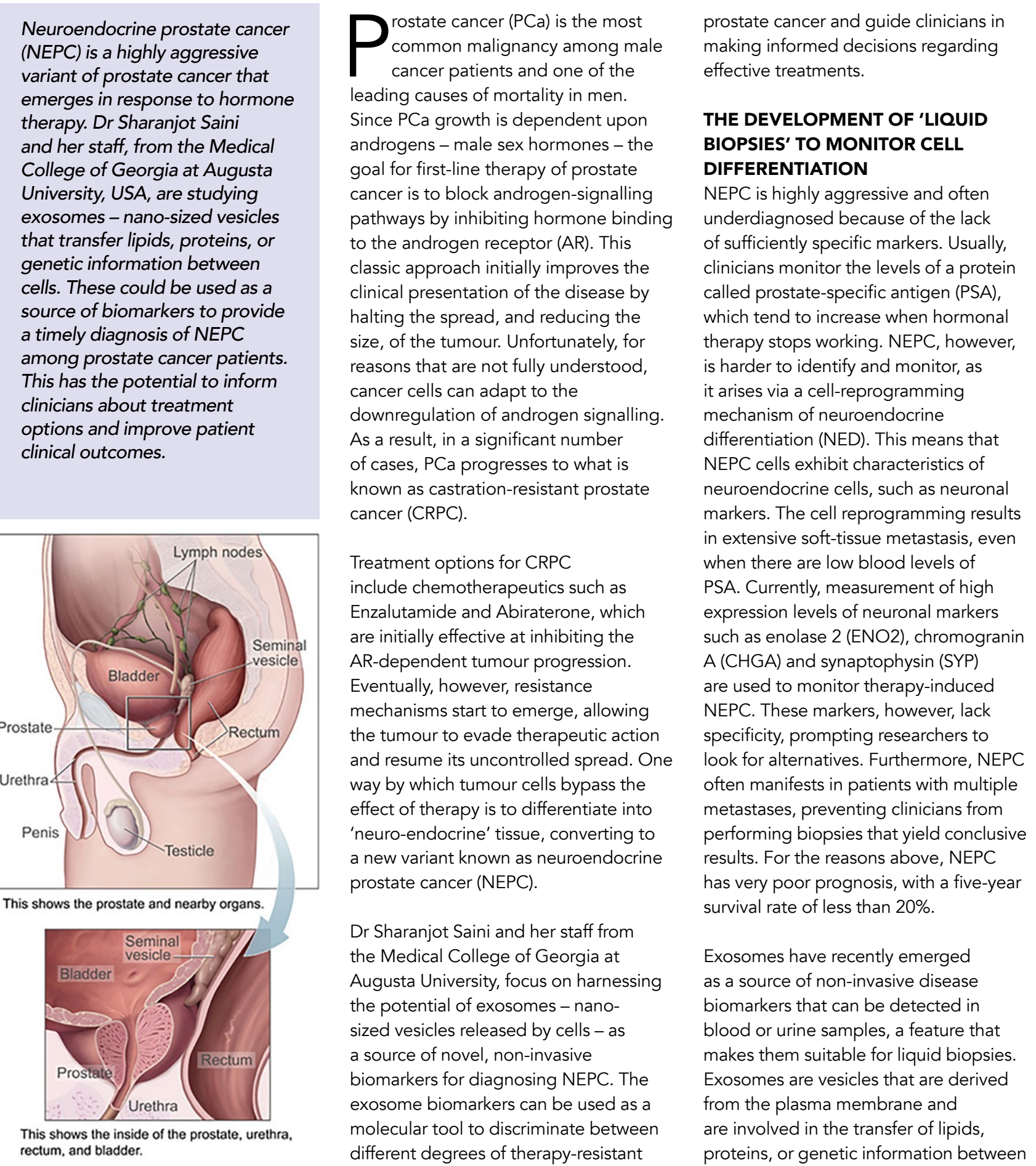

neighbouring cells. The rationale behind analysing exosome samples is that packae functional biom selectively are often reflective of the physiological and pathological state of the originating cells. The exosomes secreted by cancer cells are often involved in the cell-tocell communication pathways occurring during malignant growth and can often reveal important information about cancer stage and progression.

THE ROLE OF MICRO-RNAS N THE DEVELOPMENT OF DRUG RESISTANC

Saini and her team have previously shown that the progression of advanced CRPC is associated with a series of miRNA alterations in PCa tissues that lead to PCa gene expression pattern previous work, es well as on that conducted by different laboratories, Saini and her collaborators recently reported the identification of novel, non-invasive, exosome-based miRNA protein biomarkers for diagnosing drug-induced neuroendocrine differentiation in patients affected by prostate cancer. They published their results in late 2020, after undertaking a systematic investigation of the key miRNA alterations that are associated with the progression of advanced CRPC to therapy-induced NEPC. To explain their observations, the researchers propose that exosomal miRNAs drive Intercelluar communication, triggering cells and resulting in rapid growth cells and resulting in

For their 2020 study, Saini and her team CRPC patients and examined their

microRNA content by next generation sequencing (NGS). The genetic profiling allowed them to identify significant dysregulation of 182 known and four novel miRNAs in the exosomes of tumour tissues undergoing neuroendocrine differentiation. The research also analysed the protein content of exosomes from drug-resistant prostate cancer cell line and neuroendocrine prostate cancer cell line, detecting several dysregulated proteins associated team show dhat the protein exc. Thion
Prostate cancer roadmap

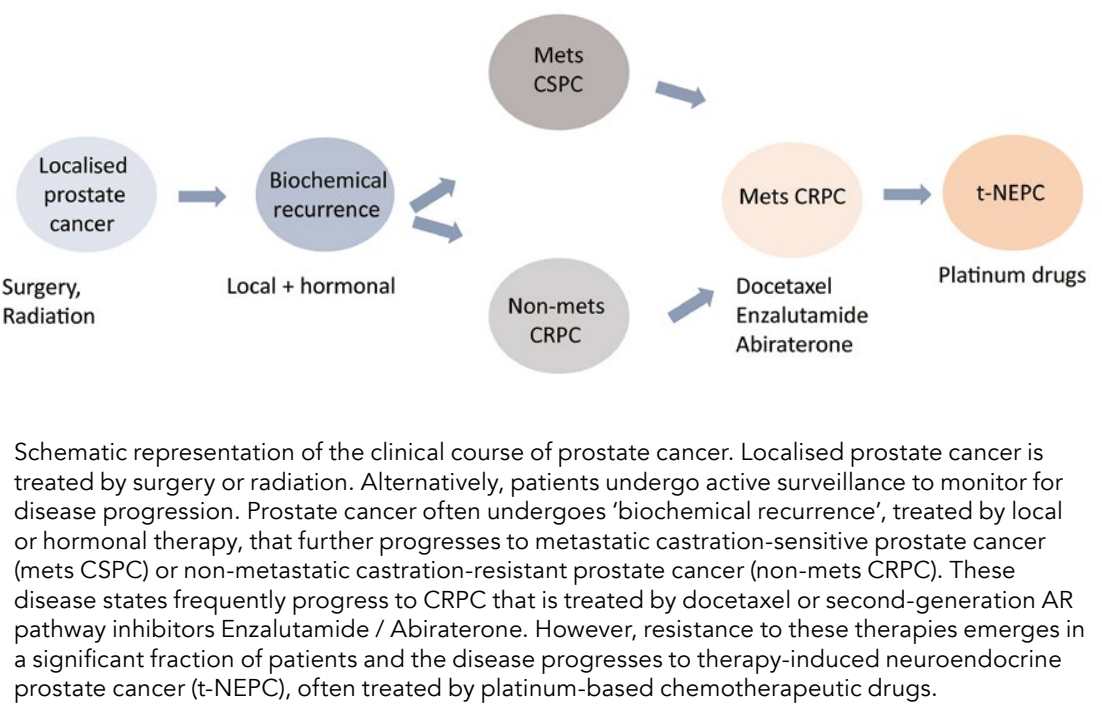

Exosome biomarkers can be used as a molecular tool to diagnose therapyresistant prostate cancer.

dysregulation affected several metabolic pathways and cellular processes, matrix organisation, and apoptosis.

CLINICAL APPLICATIONS AND FUTURE PERSPECTIVES

Understanding the molecular mechanisms behind NED is key to achieve earlier diagnosis and better prognosis for NEPC and to increase the 2020 pubstiction researchers succssullys, the the first time a characteristic set of miRNA alterations that are needed for the most aggressive form of prostate cancer to grow uncontrollably Current biomarkers are based on morphologic criteria alone and lack specificity for NEPC. In their studies, the researchers at the Saini laboratory reported for the first time a robust 'miRNA- based classifier', translatable to the clinic, for effective diagnosis of treatmentinduced NED in CRPC patients. This first-of-its-kind miRNA classifier is clearly advantageous over other molecular classifiers because of the stability and ease of detection of miRNAs. The classifier is that it can distina-based between a less-advanced state, know s adenocarcinoma, and the more ggressive neuro-endocrine carcinoma state of PCa. Saini and her team also F $N$ states that the induction correlated with androgen receptor epression and that future studies differentiation mechanisms.

The team showed, for the first time, that induction of therapy-induced with signifant aterations in miRNA and protein cargo in exosomes. These alterations can be exploited for non-invasive monitoring of therapyinduced NED in CRPC patients. Furth on increasing the numbers of CRPC neuro-endocrine samples analysed. Upon further validation, the exosome markers can be employed by clinicians for identifying cellular transformations that can lead to aggressive tumour spread in CRPC patients, providing a treatment options, and improving clinical outcomes and survival rates. The team also hopes that he miRNAs funture studies that look inted to guide novel NEPC therapeutic targets. studies will be needed with a focus more timely diagnosis of NEPC, guiding 
